\title{
Demographics, phenotypic health characteristics and genetic analysis of centenarians in China
}

\author{
Yi Zeng ${ }^{\mathrm{a}, \mathrm{b}, *}$, Qiushi Feng ${ }^{\mathrm{c}}$, Danan $\mathrm{Gu}^{\mathrm{d}}$, James W. Vaupel ${ }^{\mathrm{e}}$ \\ a Center for the Study of Aging and Human Development, Medical School of Duke University, United States \\ ${ }^{\mathrm{b}}$ Center for Healthy Aging and Development Studies, National School of Development, Raissun Institute for Advanced Studies, Peking University, China \\ ${ }^{\mathrm{c}}$ Department of Sociology \&' Centre for Family and Population Research, National University of Singapore, Singapore \\ d United Nations Population Division, United States \\ e Max Planck Institute for Demographic Research, Germany
}

\section{A R T I C L E I N F O}

\section{Article history:}

Received 9 October 2016

Received in revised form

16 December 2016

Accepted 22 December 2016

Available online $\mathrm{xxx}$

\section{Keywords:}

Centenarians

Healthy longevity

Health phenotypes

Genetic variants

GxE interactions

China

\begin{abstract}
A B S T R A C T
After a brief introduction to the background, significance and unique features of the centenarian population in China, we describe the Chinese Longitudinal Healthy Longevity Study (CLHLS), which is the world's largest study of centenarians, nonagenarians, octogenarians, and compatible young-old aged 65-79. Based on the CLHLS data and other relevant studies, we summarize demographic and socioeconomic characteristics as well as self-reported and objectively-tested health indicators of centenarians in China, with an emphasis on gender differences and rural/urban disparities. We then compare five-yearage-specific trajectories of physical and cognitive functions, self-reported health, and life satisfactions from ages 65-69 to 100+, concluding that good psychological resilience and optimism are keys to the exceptional longevity enjoyed by centenarians. We discuss recent findings of novel loci and pathways that are significantly associated with longevity based on the genome-wide association study (GWAS) of the CLHLS centenarian sample, which is 2.7 times as large as prior GWAS of longevity. We also highlight colleagues' and our own studies on longevity candidate genes and gene-environment interaction analyses. Finally, we discuss limitations inherent in our studies of centenarians in China and further research perspectives.
\end{abstract}

(c) 2016 Elsevier Ireland Ltd. All rights reserved.

\section{Contents}

1. Introduction

.00

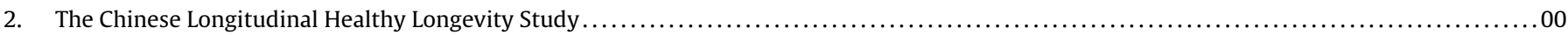

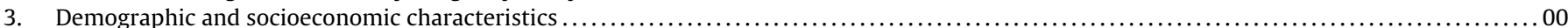

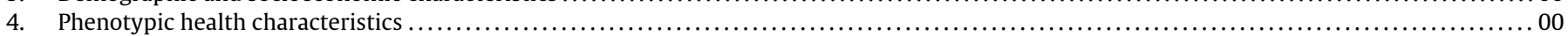

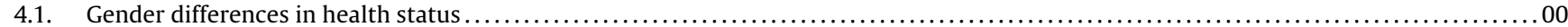

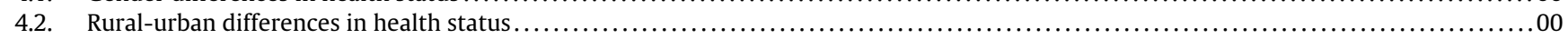

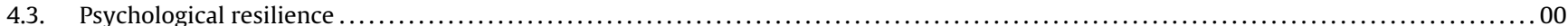

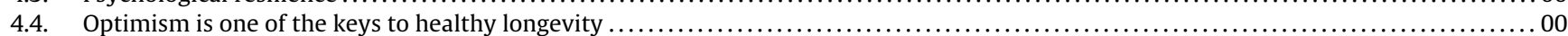

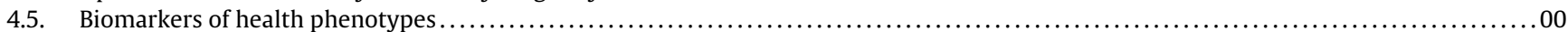

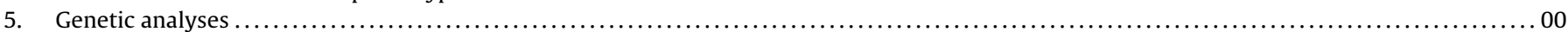

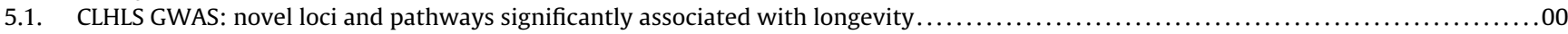

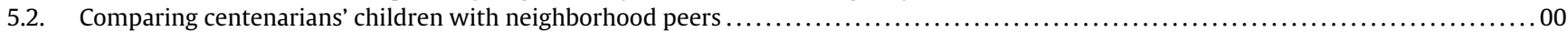

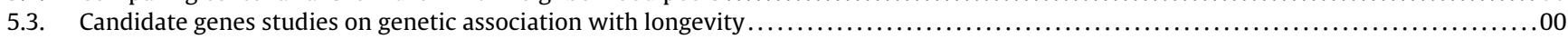

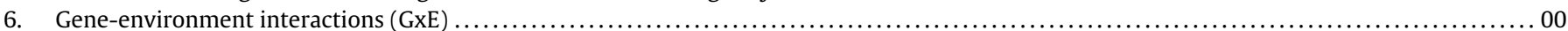

\footnotetext{
* Corresponding author at: National School of Development, Raissun Institute for Advanced Studies, Peking University, Beijing, 200871, China.

E-mail address: zengyi@nsd.pku.edu.cn (Y.Zeng).
} 


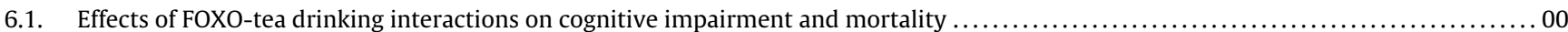

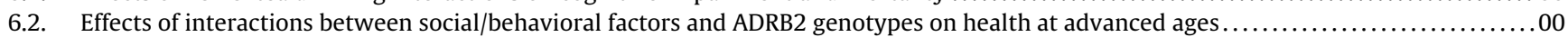

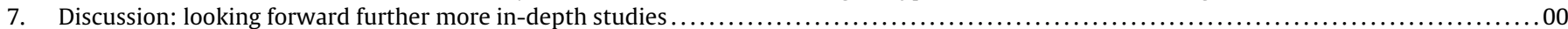

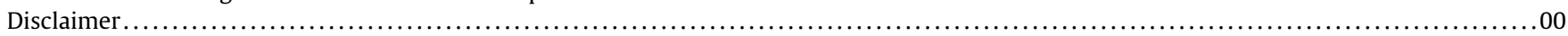

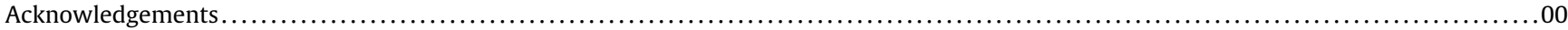

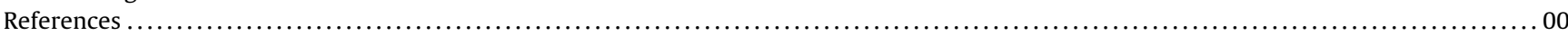

\section{Introduction}

It is widely recognized that the genomes, lifestyles (including diets) and living environments of the exceptionally long-lived population of centenarians may harbor genetic variants and social/behavioral factors associated with health and longevity (Koenig, 2001; Deelen et al., 2014; Poon and Cheung, 2012). A dozen centenarian studies have been launched in the past few decades in Europe, the U.S., and Japan. These studies have greatly improved our understanding of healthy longevity among humans (Koenig, 2001; Poon and Cheung, 2012). A general consensus in the literature is that healthy longevity is an outcome of the joint forces of socioeconomic, behavioral, environmental, and genetic factors; however, how and to what extent these factors individually, jointly and interactively contribute to centenarians' exceptionally long and healthy lives remain largely unknown. Examination on factors associated with healthy longevity, especially gene-environment interaction (GxE) effects, requires large samples. However, large scale surveys of centenarians are still rare, and with few exceptions, the sample sizes of most studies in this field are less than 1000 centenarians (Koenig, 2001; Poon and Cheung, 2012). Most centenarian studies are based on populations in industrialized countries, but it has been well acknowledged that sociodemographic, psychological, and behavioral factors and their associations with healthy longevity vary across cultural contexts and levels of social development (Poon et al., 2010). Given that $80 \%$ of the world's elderly population comes from low- and middle-income countries, it is imperative to study centenarians in less developed countries where the socioeconomic resource, healthcare service, and medical technology are comparatively disadvantaged.

Among the developing countries, China stands out as a unique case and has the largest elderly population in the world (United Nations, 2015). The relatively accurate age reporting among the Han Chinese (who accounted for $92 \%$ of the total population of China) has been well-established and re-confirmed by a wide variety of international studies; this accuracy is due to the cultural tradition of memorizing one's exact date of birth to determine important life events, such as dates of engagement, marriage, and starting to build a residential house (Coale and Li, 1991; Wang et al., 1998; Poston and Luo, 2004). There were about five centenarians per million in China in the 1990s, compared with 50 per million in Western Europe in the same period (Jeune, 1995). Consequently, Han Chinese centenarians may be more likely to have longevity-associated genes than their centenarian counterparts in the West because they survived much higher mortality regimes of the past when famine, war, and starvation operated on birth cohorts of many millions. Moreover, unlike Western countries that received many international immigrants from other parts of the world, resulting in relatively heterogeneous genetic compositions even within the same ethnic group, China has received very few international immigrants. Thus, even though one might expect population genetic substructures because of the long history of interaction with surrounding minority Chinese ethnic groups (Xu and Jin 2008), Han Chinese are relatively more homogenous in genetic composition compared to their Western counterparts (Xu et al., 2009). Clearly, the large samples of Han Chinese centenarians and other age groups for genome-wide association study (GWAS), candidate gene analyses, and GxE analyses will be instrumental for understanding determinants of healthy longevity.

In the subsequent sections of this article, we review demographic and socioeconomic characteristics, heath phenotypes, and genetic characteristics of centenarians in China. This review is mainly based on the Chinese Longitudinal Healthy Longevity Study (CLHLS), which is the world's largest study on centenarians, nonagenarians, octogenarians, and compatible young-old aged 65-79; we will also refer to the other relevant studies in the field.

\section{The Chinese Longitudinal Healthy Longevity Study}

The CLHLS has been collecting extensive data from a large sample of the oldest-old aged $80+$ including sufficient numbers of centenarians, nonagenarians and octogenarians with comparison groups of younger elders aged 65-79 since 1998. The CLHLS is conducted in a randomly selected half of the counties and cities in 22 of China's 31 provinces (Zeng, 2012), and the overwhelming majority population in the 22 provinces are Han Chinese whose age reporting is reasonably good (Coale and Li, 1991; Wang et al., 1998; Poston and Luo, 2004). The total population of the survey areas constitutes about $85 \%$ of the total population in China. So far, seven waves of data were collected in 1998, 2000, 2002, 2005, 2008, 2011, and 2014; the 8th wave data will be collected in 2017.

Within the sampled cities/counties, the CLHLS aims to interview all centenarians who voluntarily agree to participate in the study. For each centenarian interviewee, one nearby octogenarian (aged 80-89) and one nonagenarian (aged 90-99) with predefined age and sex was selected in the same city/county or neighboring city/county for voluntary participation in the CLHLS. With the same sampling strategy, three participants aged 65-79 years old were recruited for every two centenarians interviewed since the 2002 wave. Respondents who died, were lost-follow-up, or refused to be re-interviewed before or at a subsequent wave were replaced by new interviewees of the same sex and age group in the followup 2000, 2002, 2005 and 2008 waves. Table 1 shows that, over seven waves from 1998 to 2014, the CLHLS conducted 96,843 face-to-face interviews with 16,582 centenarians, 23,207 nonagenarians, 25,713 octogenarians, 20,135 younger elders aged 65-79, and 11,206 adults aged $35-64$. For the 26,242 participants who died between waves, data on mortality and degree/length of disability before death were collected in interviews with a close family member of the deceased. The CLHLS has collected DNA samples from 24,693 participants including 4849 centenarians, 5190 nonagenarians, 5274 octogenarians, 4770 young-old aged 65-79, and 4609 adults aged 40-64.

Table 2 shows that the CLHLS from 1998 to 2014 interviewed 12,047 centenarians, with 2533 males and 9514 females. These 12,047 centenarian participants produced 16,582 interviews (see Table 1); 3876 centenarians had two interviews, 1360 had three interviews, 542 had four or more interviews, and the rest had one interview. The CLHLS employed various sources to verify centenarians' ages at the individual level, including birth and marriage certificates if available; household registration booklet information; ages of their siblings, children, and relatives; genealogical records; any relevant documents from local aging committee offices if available; and reported ages in Chinese zodiac animals. 
Table 1

Age and sex structure of interviews in the seven waves of the CLHLS (1998-2014).

\begin{tabular}{|c|c|c|c|c|c|c|}
\hline \multirow[t]{2}{*}{ Age } & \multicolumn{3}{|c|}{$\begin{array}{l}\text { Number of interviews with surviving } \\
\text { participants }\end{array}$} & \multicolumn{3}{|c|}{$\begin{array}{l}\text { Number of interviews with a family member of } \\
\text { deceased participants }\end{array}$} \\
\hline & Men & Women & Total & Men & Women & Total \\
\hline $35-64$ & 7023 & 4183 & 11,206 & 4 & 2 & 6 \\
\hline $65-79$ & 10,610 & 9525 & 20,135 & 828 & 610 & 1438 \\
\hline $80-89$ & 12,860 & 12,853 & 25,713 & 2954 & 2325 & 5279 \\
\hline 90-99 & 9806 & 13,401 & 23,207 & 4383 & 5283 & 9666 \\
\hline $100+$ & 3401 & 13,181 & 16,582 & 2159 & 7694 & 9853 \\
\hline Total & 43,700 & 53,143 & 96,843 & 10,328 & 15,914 & 26,242 \\
\hline
\end{tabular}

Table 2

Number of centenarian participants by age and sex in the seven waves of the CLHLS (1998-2014).

\begin{tabular}{llll}
\hline & Men & Women & Two-sex \\
\hline 100 & 1023 & 3410 & 4433 \\
101 & 705 & 2559 & 3264 \\
102 & 386 & 1579 & 1965 \\
103 & 175 & 840 & 1015 \\
104 & 108 & 472 & 580 \\
$100-104$ & 2397 & 8860 & 11,257 \\
$105-109$ & 132 & 580 & 712 \\
$110+$ & 4 & 74 & 78 \\
Total & 2533 & 9514 & 12,047 \\
\hline
\end{tabular}

The CLHLS also verified the accuracy of age reporting at the aggregate level by using indicators such as the single-age distribution of the centenarians, the age progressive ratio among very old adults (e.g., aged 90 years or older), and centenarian density among the oldest-old, in comparison with indices from countries with good quality of data. These comparisons showed that, although the quality of age reporting of Han Chinese centenarians was not as good as in Sweden, Japan, England and Wales, it was almost as good as in Australia and Canada, slightly better than the average in the US, and much better than in Chile (Zeng and Gu, 2008). Another approach to verify the accuracy of age reporting at the population level is to check whether the age-sex-specific death rates follow a general age-sex trajectory pattern commonly found in other populations with good quality of data. The analysis by Gu and Dupre (2008) revealed that the single-age-sex-specific mortality rates at oldest-old ages including centenarians fit well with the Kannisto model, a function that is evidenced to best fit human mortality trajectories at oldest-old ages in multiple countries with high quality data (Thatcher et al., 1998). These results support the conclusion that the accuracy of age-reporting of centenarians in the CLHLS is reasonably good (Gu and Dupre, 2008).

The CLHLS questionnaire was initially translated from the instruments of the internationally well-known Danish healthy longevity survey, adapted to the Chinese cultural and socioeconomic context and carefully tested by pilot studies. Thus, the CLHLS data are internationally comparable with the data from other countries. The CLHLS collects extensive data on demographics, socioeconomic conditions, psychological traits, health practices, and health conditions. All data are collected via face-to-face interviews during in-home visits. The interviews and basic physical capacity tests are performed by a local doctor, a nurse, a local aging network worker, or a university student. The CLHLS has documented good data quality, including assessments of mortality rates, age reporting, proxy response, non-response rates, sample attrition, reliability and validity of major health measures, and rates of logically inconsistent answers (Gu, 2008; Goodkind, 2009; Chen, 2010).

\section{Demographic and socioeconomic characteristics}

Table 3 presents data on basic demographic and socioeconomic characteristics of Chinese centenarians interviewed in the CLHLS 2008 wave, which included both follow-ups and new recruitments to replace deceased and lost-follow-up interviewees at the nationwide scale, while the CLHLS 2011 and 2014 waves conducted nationwide follow-ups but had new recruitments in eight longevity areas only. The results show that a large majority ( $87.7 \%$ for males and $98.1 \%$ for females) of the centenarians were widowed and male centenarians were much more likely to be currently married (11.8\%) than their female counterparts (1.4\%). A large majority of the Chinese centenarians lived with their family members.

Living in an institution (nursing home) was uncommon, but the percentage in urban areas was 5.3 times as high as that in rural areas. Rural centenarians were relatively more likely to live alone (9.7\%) than their urban counterparts (4.9\%), likely due to family members' migration to cities and much poorer nursing home facilities in rural areas. About $55.5 \%$ of female centenarians and $29.8 \%$ of the male centenarians got married for the first time before age 20. Nearly half of this cohort of Chinese centenarians had at least 5 children and $3.5 \%$ of the centenarians had 10 or more children.

The percentage of female centenarians with no schooling was 88.6 in urban areas and 96.1 in rural areas, in sharp contrast to 48.9-64.0 for their male counterparts. About $72.9 \%$ of female centenarians and $61.9 \%$ of the male centenarians earned their living before age 60 in agriculture, husbandry, and fishery, while $19.1 \%$ of female centenarians were housekeepers. About $27.7 \%$ of the Chinese male centenarians were professionals, technicians, administrative managers or clerks before age 60 , but the corresponding figure was only $3.9 \%$ among Chinese female centenarians. The data clearly show much higher socioeconomic status throughout the life course of male centenarians compared to their female counterparts in China.

\section{Phenotypic health characteristics}

\subsection{Gender differences in health status}

The CLHLS revealed substantial advantages in self-reported health status measures among male centenarians compared to their female counterparts. Table 4 shows that male centenarians were more likely to report "good health" (56\%) than their female counterparts $(50.6 \%)$. The percentage of male centenarians who reported good life satisfaction was also slightly higher than their female counterparts. A substantial gender difference was evident in that $51.0 \%$ of male centenarians were ADL active compared to $46.4 \%$ of female centenarians.

Table 5 presents distributions of objective indicators of phenotypic health characteristics of centenarians. About $43.3 \%$ of male centenarians had "normal" or "excellent" cognitive functional capacity measured by Mini Mental State Examination (MMSE) scores, while only $25.4 \%$ of female centenarians enjoy such good 
Table 3

Percentage distributions of basic demographic and socioeconomic characteristics of Chinese centenarians by sex and rural/urban status, the CLHLS (2008).

\begin{tabular}{|c|c|c|c|c|c|c|c|c|c|}
\hline & \multicolumn{3}{|c|}{ Rural areas } & \multicolumn{3}{|c|}{ Urban areas } & \multicolumn{3}{|c|}{ Rural-Urban combined } \\
\hline & Men & Women & Two-sex & Men & Women & Two-sex & Men & Women & Two-sex \\
\hline Sample size & 369 & 1751 & 2120 & 319 & 974 & 1293 & 688 & 2725 & 3413 \\
\hline \multicolumn{10}{|l|}{ Marital status } \\
\hline Married & 10.57 & 1.26 & 2.88 & 13.17 & 1.75 & 4.56 & 11.77 & 1.43 & 3.52 \\
\hline Divorced & 0.27 & 0.06 & 0.09 & 0.00 & 0.10 & 0.08 & 0.15 & 0.07 & 0.09 \\
\hline Widowed & 88.89 & 98.40 & 96.75 & 86.21 & 97.43 & 94.66 & 87.65 & 98.06 & 95.96 \\
\hline Never married & 0.27 & 0.29 & 0.28 & 0.63 & 0.72 & 0.70 & 0.44 & 0.44 & 0.44 \\
\hline Total & 100 & 100 & 100 & 100 & 100 & 100 & 100 & 100 & 100 \\
\hline \multicolumn{10}{|l|}{ Living arrangement } \\
\hline Living with family & 88.62 & 89.21 & 89.10 & 90.91 & 88.09 & 88.79 & 89.68 & 88.81 & 88.98 \\
\hline Living alone & 10.84 & 9.48 & 9.72 & 5.33 & 4.72 & 4.87 & 8.28 & 7.78 & 7.88 \\
\hline Living in an institution & 0.54 & 1.31 & 1.18 & 3.76 & 7.19 & 6.34 & 2.03 & 3.41 & 3.14 \\
\hline Total & 100 & 100 & 100 & 100 & 100 & 100 & 100 & 100 & 100 \\
\hline \multicolumn{10}{|l|}{ Age at first marriage } \\
\hline$<=14$ & 0.89 & 6.96 & 5.89 & 0.00 & 3.18 & 2.38 & 0.47 & 5.58 & 4.53 \\
\hline $15-19$ & 29.67 & 52.59 & 48.57 & 28.99 & 45.33 & 41.22 & 29.35 & 49.94 & 45.71 \\
\hline $20-24$ & 36.20 & 33.99 & 34.38 & 41.69 & 45.23 & 44.33 & 38.82 & 38.10 & 38.25 \\
\hline $25-29$ & 17.80 & 4.68 & 6.99 & 17.59 & 4.61 & 7.88 & 17.70 & 4.66 & 7.34 \\
\hline$>=30$ & 15.43 & 1.77 & 4.17 & 11.73 & 1.65 & 4.19 & 13.66 & 1.73 & 4.18 \\
\hline Total & 100 & 100 & 100 & 100 & 100 & 100 & 100 & 100 & 100 \\
\hline Mean age at 1 st marriage & 23.08 & 19.11 & 19.81 & 22.61 & 19.67 & 20.41 & 22.86 & 19.32 & 20.04 \\
\hline \multicolumn{10}{|l|}{ Number of children ever born } \\
\hline 0 & 2.19 & 1.83 & 1.89 & 2.20 & 4.03 & 3.58 & 2.20 & 2.61 & 2.53 \\
\hline $1-2$ & 16.99 & 17.11 & 17.08 & 22.33 & 22.93 & 22.78 & 19.47 & 19.18 & 19.24 \\
\hline $3-4$ & 27.67 & 30.09 & 29.67 & 33.65 & 32.64 & 32.89 & 30.45 & 31.00 & 30.89 \\
\hline $5-6$ & 33.70 & 29.86 & 30.53 & 25.79 & 23.76 & 24.26 & 30.01 & 27.69 & 28.16 \\
\hline $7-9$ & 16.99 & 16.76 & 16.80 & 14.47 & 13.74 & 13.92 & 15.81 & 15.68 & 15.71 \\
\hline$>=10$ & 2.47 & 4.35 & 4.02 & 1.57 & 2.89 & 2.57 & 2.05 & 3.83 & 3.47 \\
\hline Total & 100 & 100 & 100 & 100 & 100 & 100 & 100 & 100 & 100 \\
\hline \multicolumn{10}{|l|}{ Number of years of schooling } \\
\hline 0 & 64.03 & 96.06 & 90.51 & 48.89 & 88.60 & 78.83 & 57.04 & 93.41 & 86.11 \\
\hline $1-2$ & 9.81 & 1.71 & 3.12 & 9.52 & 2.90 & 4.53 & 9.68 & 2.14 & 3.65 \\
\hline $3-4$ & 12.26 & 1.26 & 3.16 & 13.97 & 3.01 & 5.70 & 13.05 & 1.88 & 4.12 \\
\hline $5-6$ & 7.63 & 0.51 & 1.75 & 15.56 & 3.01 & 6.09 & 11.29 & 1.40 & 3.39 \\
\hline $7-9$ & 4.36 & 0.40 & 1.09 & 4.44 & 1.14 & 1.95 & 4.40 & 0.66 & 1.41 \\
\hline $10-12$ & 1.36 & 0.06 & 0.28 & 3.17 & 0.83 & 1.41 & 2.20 & 0.33 & 0.71 \\
\hline$>=13$ & 0.54 & 0.00 & 0.09 & 4.44 & 0.52 & 1.48 & 2.35 & 0.18 & 0.62 \\
\hline Total & 100 & 100 & 100 & 100 & 100 & 100 & 100 & 100 & 100 \\
\hline Mean \# years of schooling & 1.57 & 0.14 & 0.39 & 2.94 & 0.58 & 1.16 & 2.20 & 0.29 & 0.68 \\
\hline \multicolumn{10}{|l|}{ Occupation before age 60} \\
\hline Professionals and technicians & 4.08 & 0.17 & 0.85 & 8.78 & 1.44 & 3.25 & 6.26 & 0.62 & 1.76 \\
\hline Administrative managers & 1.63 & 0.11 & 0.38 & 3.45 & 0.10 & 0.93 & 2.47 & 0.11 & 0.59 \\
\hline Clerks & 5.43 & 1.03 & 1.79 & 34.48 & 7.09 & 13.85 & 18.92 & 3.19 & 6.36 \\
\hline Self-employed & 1.63 & 0.40 & 0.61 & 3.76 & 2.47 & 2.79 & 2.62 & 1.14 & 1.44 \\
\hline Agriculture, husbandry, fishery & 80.43 & 86.01 & 85.04 & 40.44 & 49.43 & 47.21 & 61.86 & 72.94 & 70.71 \\
\hline Houseworkers & 2.99 & 10.79 & 9.44 & 3.45 & 34.02 & 26.47 & 3.20 & 19.09 & 15.89 \\
\hline Military personnel & 0.82 & 0.29 & 0.38 & 2.19 & 0.10 & 0.62 & 1.46 & 0.22 & 0.47 \\
\hline Never worked & 0.00 & 0.57 & 0.47 & 0.63 & 3.60 & 2.86 & 0.29 & 1.65 & 1.38 \\
\hline Others & 2.99 & 0.63 & 1.04 & 2.82 & 1.75 & 2.01 & 2.91 & 1.03 & 1.41 \\
\hline Total & 100 & 100 & 100 & 100 & 100 & 100 & 100 & 100 & 100 \\
\hline
\end{tabular}

cognition. Male centenarians were more likely to be able to stand up from the chair without using hands (41.1\%) than their female counterparts (32.0\%). Similar substantial male advantages were observed in other physical performance tests of picking up a book from the floor and turning around $360^{\circ}$. Male centenarians were much more likely to see things and distinguish the direction (39\%) than their female counterparts (29.5\%). About $46.4 \%$ of female centenarians could not see things clearly or were even blind, in contrast to $38.8 \%$ of male centenarians. Female centenarians were also much more likely to live without any teeth $(62.5 \%)$ than their male counterparts (51.6\%). Clearly, Chinese male centenarians experience substantial advantages in self-reported health, ADL ability, objectively-tested cognitive capacity, physical performance, visual function, and residual teeth, compared to their female counterparts.

The gender differences in health profiles presented in Tables 4-5 and outlined above are consistent with the gender health-survival paradox that women tend to live longer but experience worse health compared to men (Van Oyen et al., 2013) and with find- ings of previous studies using data from the other waves of CLHLS (Zeng et al., 2002; Zeng et al., 2003; Zhang 2006), most recent oldest-old cohorts comparative analysis using the CLHLS data (Zeng et al., 2017), and other studies of Chinese elders (Wang et al., 2009). The gender paradox is often related to gender disparity in socioeconomic status, genetic and acquired risks, and disease patterns and prevention. Regarding Chinese centenarians, the large gender differences in health are possibly related to male advantages in socioeconomic status and more outdoor physical activities, such as gardening, farming and fishing, which facilitate maintenance of capacity for daily living and physical performance. Similar gender patterns were also found among French centenarians and explained by social and behavioral differences (Balard et al., 2011). Another possible explanation of the large gender difference in health indicators among Chinese centenarians is that the male centenarians are much more stringently selected "longevity-stars" due to higher death rates in males than in females at all younger ages. 
Table 4

Percentage distributions of self-reported indicators of health and life satisfaction of Chinese centenarians by sex and rural/urban status, the CLHLS (2008).

\begin{tabular}{|c|c|c|c|c|c|c|c|c|c|}
\hline & \multicolumn{3}{|c|}{ Rural areas } & \multicolumn{3}{|c|}{ Urban areas } & \multicolumn{3}{|c|}{ Rural-Urban combined } \\
\hline & Men & Women & Two-sex & Men & Women & Two-sex & Men & Women & Two-sex \\
\hline Sample size & 369 & 1751 & 2120 & 319 & 974 & 1293 & 688 & 2725 & 3413 \\
\hline \multicolumn{10}{|l|}{ Self-reported health } \\
\hline Good & 54.61 & 51.09 & 51.78 & 57.61 & 49.78 & 51.87 & 56.00 & 50.61 & 51.82 \\
\hline Moderate & 30.85 & 35.05 & 34.22 & 32.92 & 37.93 & 36.59 & 31.81 & 36.11 & 35.14 \\
\hline Bad & 14.54 & 13.86 & 14.00 & 9.47 & 12.29 & 11.54 & 12.19 & 13.29 & 13.04 \\
\hline Total & 100 & 100 & 100 & 100 & 100 & 100 & 100 & 100 & 100 \\
\hline \multicolumn{10}{|c|}{ Self-reported life satisfaction } \\
\hline Good & 59.93 & 62.57 & 62.04 & 72.43 & 67.12 & 68.53 & 65.71 & 64.24 & 64.57 \\
\hline Moderate & 34.75 & 30.89 & 31.65 & 25.10 & 28.70 & 27.74 & 30.29 & 30.08 & 30.13 \\
\hline Bad & 5.32 & 6.54 & 6.30 & 2.47 & 4.19 & 3.73 & 4.00 & 5.67 & 5.30 \\
\hline Total & 100 & 100 & 100 & 100 & 100 & 100 & 100 & 100 & 100 \\
\hline \multicolumn{10}{|c|}{ Activities of Daily Living (ADL) } \\
\hline Active & 61.52 & 52.20 & 53.82 & 38.87 & 35.93 & 36.66 & 51.02 & 46.39 & 47.32 \\
\hline Mild disability & 16.53 & 21.99 & 21.04 & 36.99 & 28.95 & 30.94 & 26.02 & 24.48 & 24.79 \\
\hline Severe disability & 21.95 & 25.81 & 25.14 & 24.14 & 35.11 & 32.41 & 22.97 & 29.14 & 27.89 \\
\hline Total & 100 & 100 & 100 & 100 & 100 & 100 & 100 & 100 & 100 \\
\hline
\end{tabular}

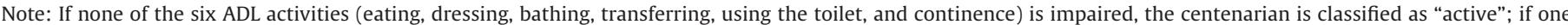

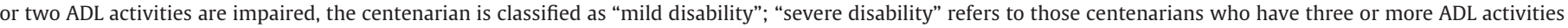
impaired.

Table 5

Percentage distributions of objective health indicators of Chinese centenarians, the CLHLS (2008).

\begin{tabular}{|c|c|c|c|c|c|c|c|c|c|}
\hline & \multicolumn{3}{|c|}{ Rural areas } & \multicolumn{3}{|c|}{ Urban areas } & \multicolumn{3}{|c|}{ Rural-Urban combined } \\
\hline & Men & Women & Two-sex & Men & Women & Two-sex & Men & Women & Two-sex \\
\hline Sample size & 369 & 1751 & 2120 & 319 & 974 & 1293 & 688 & 2725 & 3413 \\
\hline \multicolumn{10}{|l|}{ Mini Mental State Examination (MMSE) } \\
\hline Maximum & 18.87 & 10.60 & 12.18 & 17.10 & 8.53 & 10.79 & 18.04 & 9.84 & 11.64 \\
\hline Normal & 25.50 & 14.81 & 16.85 & 24.91 & 16.80 & 18.94 & 25.22 & 15.54 & 17.67 \\
\hline Mild impairment & 14.90 & 16.13 & 15.90 & 15.61 & 18.67 & 17.86 & 15.24 & 17.07 & 16.67 \\
\hline Severe impairment & 40.73 & 58.46 & 55.08 & 42.38 & 56.00 & 52.40 & 41.51 & 57.55 & 54.03 \\
\hline Total & 100 & 100 & 100 & 100 & 100 & 100 & 100 & 100 & 100 \\
\hline \multicolumn{10}{|l|}{ Ability to stand up from the chair } \\
\hline Yes, without using hands & 39.30 & 30.91 & 32.38 & 43.26 & 33.95 & 36.25 & 41.13 & 32.00 & 33.85 \\
\hline Yes, using hands & 44.44 & 48.39 & 47.69 & 37.93 & 40.53 & 39.89 & 41.42 & 45.57 & 44.73 \\
\hline Unable & 16.26 & 20.70 & 19.92 & 18.81 & 25.51 & 23.86 & 17.44 & 22.43 & 21.42 \\
\hline Total & 100 & 100 & 100 & 100 & 100 & 100 & 100 & 100 & 100 \\
\hline \multicolumn{10}{|l|}{ Ability to pick up a book from the floor } \\
\hline Yes, standing & 40.11 & 28.14 & 30.23 & 38.87 & 26.16 & 29.30 & 39.53 & 27.43 & 29.88 \\
\hline Yes, sitting & 36.59 & 41.09 & 40.30 & 28.21 & 31.82 & 30.93 & 32.70 & 37.78 & 36.75 \\
\hline Unable & 23.31 & 30.77 & 29.47 & 32.92 & 42.02 & 39.77 & 27.76 & 34.79 & 33.37 \\
\hline Total & 100 & 100 & 100 & 100 & 100 & 100 & 100 & 100 & 100 \\
\hline \multicolumn{10}{|l|}{ Ability to turn around $360^{\circ}$} \\
\hline Yes, $<=10$ steps & 44.72 & 34.89 & 36.60 & 42.01 & 27.54 & 31.11 & 43.46 & 32.27 & 34.53 \\
\hline Yes, $>10$ steps & 3.25 & 2.40 & 2.55 & 2.51 & 2.16 & 2.24 & 2.91 & 2.31 & 2.43 \\
\hline Unable & 52.03 & 62.71 & 60.85 & 55.49 & 70.30 & 66.64 & 53.63 & 65.42 & 63.04 \\
\hline Total & 100 & 100 & 100 & 100 & 100 & 100 & 100 & 100 & 100 \\
\hline \multicolumn{10}{|l|}{ Visual function } \\
\hline Can see and distinguish & 38.63 & 29.76 & 31.33 & 39.43 & 28.93 & 31.53 & 39.00 & 29.46 & 31.41 \\
\hline Can see only & 23.84 & 24.94 & 24.75 & 20.19 & 22.79 & 22.14 & 22.14 & 24.16 & 23.75 \\
\hline Can't see & 34.25 & 40.82 & 39.66 & 37.85 & 45.06 & 43.27 & 35.92 & 42.35 & 41.04 \\
\hline Blind & 3.29 & 4.47 & 4.26 & 2.52 & 3.23 & 3.05 & 2.93 & 4.02 & 3.80 \\
\hline Total & 100 & 100 & 100 & 100 & 100 & 100 & 100 & 100 & 100 \\
\hline \multicolumn{10}{|l|}{ Number of residual teeth } \\
\hline 0 & 50.14 & 61.46 & 59.49 & 53.29 & 64.44 & 61.69 & 51.60 & 62.53 & 60.32 \\
\hline $1-3$ & 16.53 & 18.47 & 18.13 & 16.61 & 15.21 & 15.56 & 16.57 & 17.30 & 17.16 \\
\hline $4-6$ & 13.55 & 10.06 & 10.67 & 13.17 & 10.89 & 11.46 & 13.37 & 10.36 & 10.97 \\
\hline $7-9$ & 6.78 & 3.49 & 4.06 & 6.27 & 3.08 & 3.87 & 6.54 & 3.34 & 3.99 \\
\hline $10-12$ & 6.50 & 2.92 & 3.54 & 3.76 & 3.19 & 3.33 & 5.23 & 3.01 & 3.46 \\
\hline $13-15$ & 1.08 & 0.97 & 0.99 & 2.19 & 1.23 & 1.47 & 1.60 & 1.07 & 1.17 \\
\hline $16-18$ & 1.90 & 1.14 & 1.27 & 1.57 & 0.31 & 0.62 & 1.74 & 0.84 & 1.03 \\
\hline$>=19$ & 3.52 & 1.49 & 1.84 & 3.13 & 1.64 & 2.01 & 3.34 & 1.54 & 1.91 \\
\hline Total & 100 & 100 & 100 & 100 & 100 & 100 & 100 & 100 & 100 \\
\hline
\end{tabular}

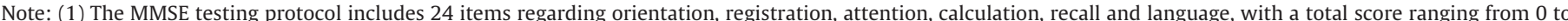

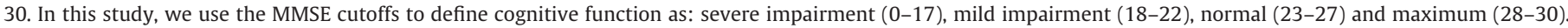

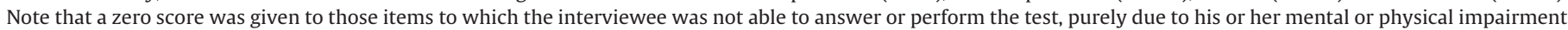

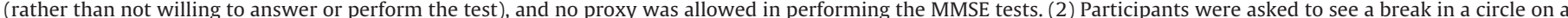

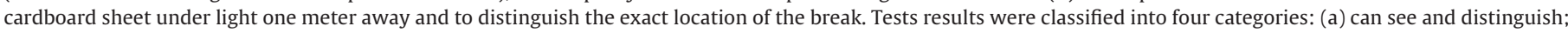
(b) can see only; (c) can't see; and (d) blind. 


\subsection{Rural-urban differences in health status}

As shown in Table 4, slightly more than half of centenarians reported "good health" with almost no rural-urban difference. However, a considerably higher proportion of centenarians in urban areas reported good life satisfaction (68.5\%) compared to their rural counterparts (62.0\%). The rural-urban differences in ADL status were remarkably large, with $53.8 \%$ of centenarians in rural areas reporting active ADL status in contrast to $36.7 \%$ of centenarians in urban areas. Table 5 presents much better physical performance in rural centenarians compared to urban counterparts, but unsubstantial rural-urban differences in MMSE scores, visual function, and residual teeth among the Chinese centenarians.

The CLHLS data clearly revealed that, compared to their urban counterparts, rural centenarians enjoyed better health status measured by self-reported ADL ability and objective physical performance tests. On the other hand, urban centenarians tended to report better life satisfaction than their rural counterparts. Why do rural centenarians enjoy better health than their urban counterparts in China? A few possible explanations may help us to understand this phenomenon. First, limited available facilities may force rural centenarians to perform daily activities by themselves; this frequent exercise may enable them to maintain their capacities for daily living and physical performance for a longer time than their urban counterparts. Furthermore, centenarians in rural areas are likely to continue to perform garden work to grow vegetables or even to perform some light labor in the fields, which may also help them to maintain their capacity for daily living and physical performance. These explanations may also help us to understand the fact that the elderly in Indonesia, Malaysia, the Philippines, and Thailand were found to be more active in daily living than the elderly in developed countries (Ju and Jones, 1989; Lamb, 1999). A study found that the ADL functional capacity of centenarians in three major Chinese cities of Beijing, Hangzhou and Chengdu was significantly stronger than that of Danish centenarians in 1997 (Wang, 2001).Second, the natural environment is likely healthier in rural areas. Industrial pollution in the cities may lessen the capacity of centenarians to maintain good health. Third, the harder life and higher mortality at younger ages in rural areas may have resulted in rural centenarians to be more robust than their counterparts in cities and towns (Zeng et al., 2002). Despite these health advantages for rural centenarians, life satisfaction has more links with social economic attainment; the centenarians living in rural areas are usually illiterate with lower socioeconomic status, which may lead to the relatively low life satisfaction among rural centenarians (Li and Wu, 2008).

\subsection{Psychological resilience}

Previous studies have demonstrated that the psychological construct of resilience is positively correlated with cognitive function, physical health, and self-reported health among the elderly (Hardy et al., 2002; Wagnild, 2003), as well as with self-rated successful aging in developed countries (Lamond et al., 2009). Poon et al. (1992) discovered that the common characteristics of the members of the Georgia centenarians sample were optimism and flexibility, which are associated with psychological resilience (Riolli et al., 2002). Selim et al. (2005) reported that U.S. centenarian veterans were psychologically resilient despite poor physical health. Jopp and Rott (2006) also demonstrated that psychological resilience was well preserved at the very end of the life span in the Heidelberg Centenarian Study.

However, most of the prior studies on this specific topic were based on small samples with limited numbers of centenarians and nonagenarians, which restricted estimation efficiency (Jopp and Rott, 2006; Pascucci and Loving, 1997). The CLHLS analysis on cen- tenarians' psychological resilience tried to expand this literature (Zeng and Shen, 2010), by adopting the simplified and straightforward measurement of psychological resilience - the ability to adapt positively to adversity - specified by Lamond et al. (2009): the simplified psychological resilience score (SPRS). The SPRS is based on the available data collected through seven questions in CLHLS (See Table 2 in Zeng and Shen, 2010) that reflect personal tenacity, optimism, coping with negative mood, secure relationships, and self-control, which are deemed as important factors of psychological resilience (Connor and Davidson, 2003). Statistical analysis showed that the psychometric properties of SRS based on the CLHLS data are acceptable (Zeng and Shen, 2010).

Based on cross-sectional data from the CLHLS 2008 wave, with a total sample size of 16,566 elderly respondents aged 65 and older (including 3413 centenarians, 4596 nonagenarians, 4272 octogenarians, and 4285 young-old aged 65-79), logistic regressions showed that, after controlling for various confounders including age, sex, education, rural/urban residence, physical health and cognitive status, centenarians were significantly more resilient than any other old-age group (Zeng and Shen, 2010). For example, centenarians were significantly more resilient than the young-old by a margin of $26.1 \%(p<0.01)$. Logistic regression analyses based on the longitudinal data showed that nonagenarians aged 94-98 with better psychological resilience had a $43.1 \%$ higher likelihood of becoming a centenarian compared to nonagenarians who were members of the same birth cohort but with lower psychological resilience $(p<0.01)$, adjusted for various confounding factors including health status. These results suggest that psychological resilience significantly contributes to longevity at all ages and it becomes even more pronounced at very advanced ages to achieve exceptional longevity (Zeng and Shen, 2010). This is consistent with another study based on the CLHLS longitudinal data which showed that, on average, better psychological resilience significantly reduced mortality risk by about $15.5 \%$ among all elderly adults aged $65+$, adjusted for various confounding factors including age, sex, rural/urban residence, physical and mental health (Shen and Zeng, 2010).

\subsection{Optimism is one of the keys to healthy longevity}

Fig. 1 based on the CLHLS 2008 data shows that the proportion of elders that are active in daily living, have good physical performance capacity, and have normal cognitive functioning dropped dramatically from ages 65-69 to $100+$. The percentage of selfreported life satisfaction and self-reported good health, however, remained almost constant or even slightly increased from age 65-69 to 100+. The interesting pattern depicted in Fig. 1 has been repeatedly reconfirmed by our multi-wave CLHLS datasets (Zeng and Vaupel, 2002; Zeng, 2012) and it reveals that long-lived people, especially centenarians, likely maintain stable life satisfaction independent of their capacities in daily activities, physical performance, and cognitive function.

In the U.S., a longitudinal study in Berkeley found that life satisfaction does not decrease until advanced old age (Field and Millsap, 1991). Based on a survey of 3998 elders aged 65+ in the U.S., Blazer et al. (1991) indicated that the "oldest-old" suffer fewer depressive symptoms compared to younger age groups when other confounding variables such as age, gender, income, physical disability, cognitive impairment, and social support are taken into account. Our present findings, based on an unprecedented large data set of the oldest-old population including centenarians in a developing country, are consistent with these previous studies.

These findings may suggest that optimism is one of the secrets of longevity, as optimistic long-lived centenarians and nonagenarians are more likely to look positively into the future, think optimistically, and thus view their life as "satisfactory". The old Chinese 


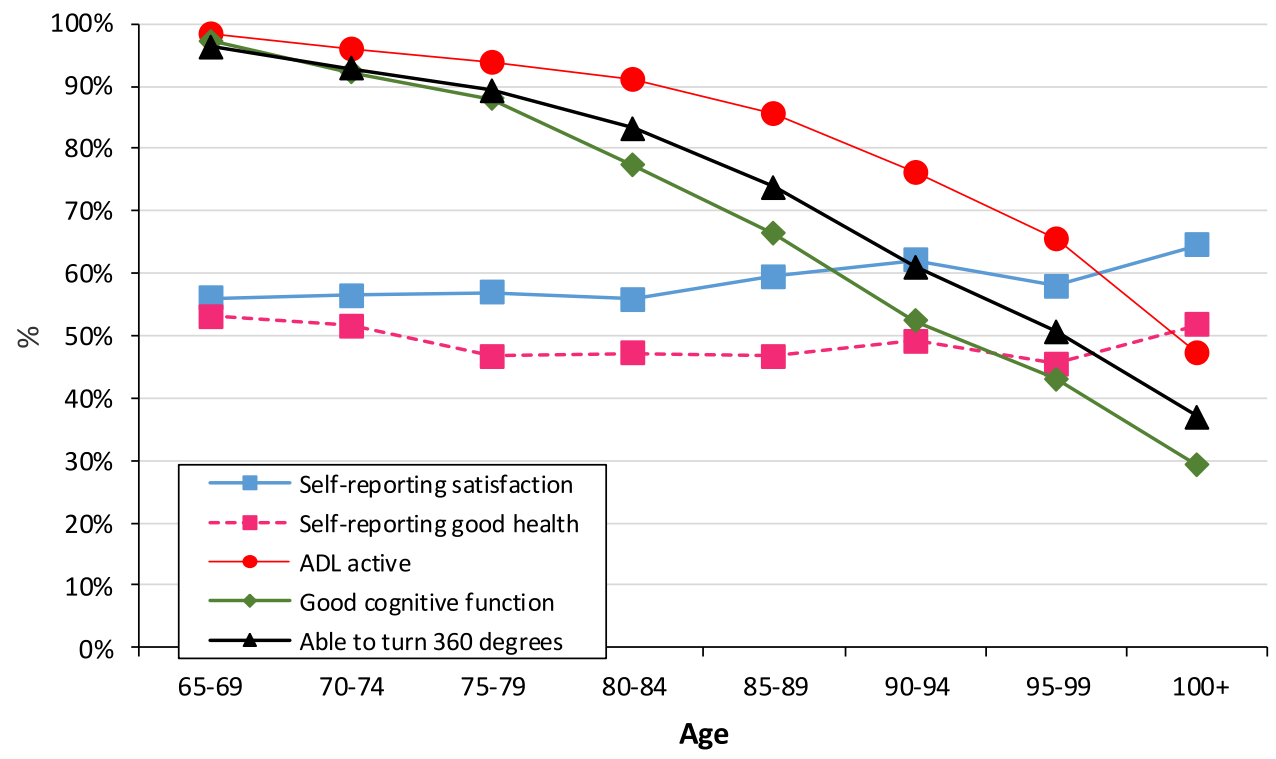

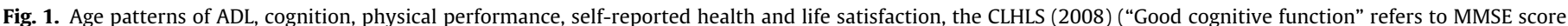
$\geq 23$ ).

saying "Knowing satisfaction leads to constant happiness" (Zhi Zu Chang Le) illustrates the connection between life satisfaction and happiness, which may promote longevity (Zeng and Vaupel, 2002).

\subsection{Biomarkers of health phenotypes}

As clinical components of epidemiological surveys and simple in-home collection of bodily fluids become more available, it is increasingly important to study biomarkers of health phenotypes. Based on 60 longevity families (206 subjects, consisting of 61 centenarians and their first and second generation of offspring with their spouses), the research group led by Yaping Zhang and Qingpeng Kong found that serum lipids, kidney function, and diastolic pressure rather than systolic pressure were more favorable among centenarians and their offspring, suggesting that these factors may play an important role in familial longevity (He et al., 2016). Yin et al. (2012) found that, compared with the younger elderly, centenarians had lower levels of diastolic blood pressure (DBP) and triglycerides (TG), higher levels of total cholesterol (TC) (but in the normal range), and higher high-density lipoprotein (HDL) levels, which meant that the centenarians had good blood lipid profiles and thus had low risk of cerebro-vascular disease. Studies also showed that the predictive value of blood lipid profiles for cardiovascular diseases declined after the age of 75 and low levels of TC were associated with higher mortality (Spada et al., 2007), which also meant higher levels of TC in the normal range may be helpful for 'healthy aging'. All in all, maintaining lower DBP and good blood lipid profile are important characteristics of centenarians (Yin et al., 2012). Based on a sample of 107 centenarians and 18 great-grandchildren of these centenarians, the research group led by Wuyi Wang and Yonghua Li found that sufficient $\mathrm{Zn}$ and Se concentrations as well as low exposure to heavy metals pollution contributed to exceptional longevity (Li et al., 2011). This group's follow-up study on 78 centenarians suggests that regulating in vivo contents of trace elements, especially $\mathrm{Se}, \mathrm{Co}$, and $\mathrm{Zn}$, is a reasonable approach to intervene with geriatric diseases (Li et al., 2012). Based on a sample of 255 centenarians and their 124 children and grandchildren, this group also found that adequate concentrations of $\mathrm{Fe}$ and Se might increase the life span of centenarians, whereas excessive concentrations of $\mathrm{P}$ and $\mathrm{Pb}$ were harmful to health and may reduce life span (Hao et al., 2016).

\section{Genetic analyses}

\subsection{CLHLS GWAS: novel loci and pathways significantly} associated with longevity

As reviewed in the introduction section, centenarian genomes may harbor genetic variants associated with health and longevity (Deelen et al., 2014). In recent years, a number of genome-wide association studies (GWAS) on longevity in North America and Europe have been conducted, but only two loci have been identified as being associated with longevity at a genome-wide significant level: the well-known TOMM40/APOE/APOC1 locus which is negatively associated with longevity (Newman et al., 2010; Sebastiani et al., 2012; Deelen et al., 2014; Broer et al., 2015), and a second locus on chromosome $5 q 33.3$, which was identified in a recent GWAS by Deelen et al. (2014).

The largest sample size of centenarians in reported GWAS studies before publication of the CLHLS GWAS on longevity was 801 , in which the study found one genome-wide significant single nucleotide polymorphism (SNP) (Sebastiani et al., 2012). While interest in this topic is strong, the field is hindered by a lack of databases with both genotypic and phenotypic information and sufficiently large sample sizes of centenarians; much larger samples of centenarians and ethnically matched controls are needed for genome-wide significant discoveries of genetic associations with longevity (Sebastiani et al., 2015; Newman et al., 2010). To expand the catalogue of longevity-associated loci and gain a better understanding of the influences of genes and biological pathways on longevity, the CLHLS team performed a GWAS using samples including 2178 Han Chinese centenarians, which is about 2.7 times the largest sample size of previously published GWAS on centenarians (Sebastiani et al., 2012), and 2299 middle-aged controls, which is of reasonably good power (Zeng et al., 2016a). This work also represents the first GWAS of longevity in Asian populations and in developing countries more generally.

The CLHLS GWAS of Han Chinese centenarians and middleaged controls produced a few novel findings. First, it identified 11 independent loci associated with longevity and replicated in two independent GWAS datasets from Southern and Northern regions of China (Table 1, Zeng et al., 2016a). More specifically, it identified two novel loci (rs2069837-IL6 and rs2440012-ANKRD20A9P) 
that reached genome-wide significance, plus 9 loci with suggested significance $\left(\mathrm{P}<3.64 \times 10^{-5}\right)$, including rs405509 in APOE, which is a well-known locus negatively associated with longevity (Rebeck et al., 1994). The strongest novel signal is for the rs2069837 SNP $\left(\mathrm{P}=1.80 \times 10^{-9}\right)$ within the IL6 locus at $7 \mathrm{p} 15.3$, which is negatively associated with longevity in the Han Chinese; this is consistent with previous findings that the IL6 gene functions as an inflammatory biomarker of poor health outcomes (Bonafè et al., 2001; Cohen et al., 2003) and is associated with longevity (Soerensen et al., 2013). Another strong novel locus that reached genome-wide significance in Han Chinese GWAS was ANKRD20A9P (ankyrin repeat domain 20 family, member A9), a pseudogene which is affiliated with the IncRNA class.

The CLHLS GWAS also identified 8 independent SNPs associated with longevity with $\mathrm{P}<10^{-4}$ in Han Chinese that overlapped results from the European longevity GWAS (Deelen et al., 2014) and/or New England longevity GWAS (Sebastiani et al., 2012), with at least nominal significance $(P<0.05)$. It also confirmed 4 previously reported longevity-associated SNPs and identified 2 independent associations with longevity in the well-known TOMM40/APOE/APOC1 LD region. The findings provided additional new signals and replicated the negative association of the TOMM40/APOE/APOC1 LD region with exceptional longevity in Han Chinese, and confirmed 5q33.3 as a longevity locus that was previously identified in the European longevity GWAS (Deelen et al., 2014). In short, the CLHLS GWAS demonstrated both similarities and differences in genetic associations with longevity across continents and ethnicities.

At the pathway level, the CLHLS GWAS study identified 4 major pathways that influence longevity, including starch, sucrose and xenobiotic metabolism; immunity; MAPK signaling; and calcium homeostasis. Among these pathways, p38 MAPK and immunity have been previously related to longevity in numerous studies (Asai et al., 2002; Naumova et al., 2011; Yao et al., 2015). Network analysis indicated that these 4 pathways are highly interconnected. Collectively, these findings strongly support the notion that longevity is a polygenic trait influenced by a complex interplay of multiple genes and pathways. Interestingly, the three significant pathways (MAPK, immunity, and calcium signaling) identified in the Han Chinese GWAS study were also most recently reconfirmed as significant pathways in a Danish longitudinal epigenome-wide association study (Tan et al., 2015). The novel finding that the starch, sucrose and xenobiotic metabolism pathway is significantly associated with human longevity in Han Chinese is consistent with a previous animal model study in Drosophilia (Troen et al., 2007).

The findings from the CLHLS GWAS study support the hypothesis that defensive mechanisms (such as immunity) and metabolism driven by diet in response to environmental stress may play key roles in longevity of the Han Chinese. Diet mediated mechanisms also suggest that genetic and epigenetic influences upon extreme survival can differ according to culture and ethnicity. These findings may provide novel insights for expanding longevity theories that had emphasized genes associated with maintenance of genome integrity (especially DNA repair) and fertility-related mechanisms (Kirkwood and Kowald, 2012; Baudisch and Vaupel, 2012; Sun et al., 2015).

\subsection{Comparing centenarians' children with neighborhood peers}

A comparative analysis between centenarians' children (familial longevity) and neighborhood peers (controls) is an efficient approach to learn how genetic inheritance and family lifestyle and their interaction with environmental factors affect healthy aging. For example, prior research has suggested that centenarian offspring have better profiles of blood pressure and diabetes mellitus compared to controls (Barzilai et al., 2001); similarly, Adams et al.
(2008) found that centenarian offspring had a 78\% lower risk of myocardial infarction, $83 \%$ lower risk of stroke, and 86\% lower risk of developing diabetes mellitus than the referent group. However, all studies comparing centenarians' offspring and their peers based on data prior to the CLHLS (Zeng et al., 2012) were from industrialized countries. Moreover, prior studies in this field investigated a limited number of specific diseases and disorders, but none of them dealt with multiple dimensions of physical health, mental health and subjective well-being simultaneously in a single study, and they did not explore the effects of interactions between familial longevity and environment on health outcomes. The CLHLS study on centenarians' children and neighborhood peers intended to fill these research gaps by exploring the effects of familial longevity influence and its interactions with several environmental factors on physical health, mental health, and subjective well-being at old ages (Zeng et al., 2012).

Zeng et al. (2012) conducted multivariate regression analysis, employing the unique CLHLS data from 417 children (mean age 69.0) of centenarians and 560 neighborhood peers (mean age 71.0) who have no family history of longevity. It was found that, compared to the neighborhood peers and adjusted for age, gender and six major environmental factors, the centenarians' children had significantly better instrumental activities of daily living (IADL) function $(\mathrm{p}<0.001)$, smaller number of chronic conditions or health problems $(p<0.01)$, less anxiety and loneliness $(p<0.01)$, better cognitive function $(\mathrm{p}<0.01)$, more psychological resilience $(p<0.01)$, better self-rated health $(p<0.001)$, and better self-rated life satisfaction $(p<0.001)$. Based on these findings, we conclude that familial longevity is strongly and positively associated with physical health, mental health, and subjective well-being at old ages.

The results also revealed that the interactions between familial longevity and any one of the three environmental factors (receipt of adequate medical care when ill as a child, number of living children, and household economic conditions) were significantly associated $(\mathrm{p}<0.05$ ) with the three health outcome indicators (IADL, self-rated life satisfaction, and anxiety-loneness) at old ages. We discovered that the effects of these environmental factors on the health outcome indicators were substantially stronger among elders who had no family history of longevity compared to centenarians' children who likely carry genes and/or inherited healthy behavior and better lifestyle from long-lived parents. This interesting finding may be understood from a more general mechanism: in the absence of the ascribed protective factor (e.g., familial longevity influence), the other protective factors have stronger effects on health. This finding, although it mixes genetic inheritance and family lifestyle, is generally consistent with some other studies focusing on effects of interactions between genetic variants and behavioral/environmental factors. For example, Zeng et al. (2011) found that the negative effects of four life stress factors on self-rated health among carriers of APOE4, a genotype which is associated with poor health and higher mortality (Blazer et al., 2001), are much stronger than that among APOE4 non-carriers (Zeng et al., 2011). These findings imply that, although familial longevity is one of the important factors which determine health outcome, socioeconomic, behavioral, and environmental factors may be significantly more important for those who do not have the advantage of family history of longevity. Thus, when considering cost-benefit effectiveness of health promotion programs and the greater health assistance needs for disadvantaged groups, future interventions may need to target those who do not have the advantage of familial longevity (Ryff and Singer, 2005). 


\subsection{Candidate genes studies on genetic association with longevity}

The research group led by Xiaoli Tian and Yi Zeng tested the genetic contribution of FOXO1A and FOXO3A to the longevity phenotype in the Han Chinese population based on CLHLS samples from 1817 centenarians and younger individuals collected in 1998 (Li et al., 2009; Zeng et al., 2010). Among the six selected and genotyped tagging SNPs from the FOXO1A and FOXO3A genes, two SNPs of FOXO1A were found to be associated with longevity in women $(p=0.01-0.005)$, whereas all three SNPs of FOXO3A were associated with longevity in both genders $(p=0.005-0.001)$. The association of FOXO1A with female longevity was replicated in 700 centenarians and younger individuals that were sampled from geographically different areas than the original population. Thus, we demonstrated that, unlike FOXO3A, FOXO1A is more closely associated with female longevity, suggesting that the genetic contribution to longevity trait may be moderated by gender (Li et al., 2009). This research group also tested whether genes encoding for key components of the $\beta$ adrenergic signaling pathway are associated with human longevity, based on CLHLS samples of 963 centenarians and 1028 geographymatched young individuals. This study indicated that enhanced production of $\beta 2$-adrenergic receptors caused by genetic variants is inversely associated with human longevity (Zhao et al., 2012).

Based on a sample of 338 centenarians, 86 nonagenarians, and 537 controls aged 40-70, the research group led by Yaping Zhang and Qingpeng Kong found that higher mitochondrial DNA content may convey a beneficial effect to exceptional longevity through assuring sufficient energy supply (He et al., 2014). This group discovered that the absence of A673T variant in the amyloid- $\beta$ precursor protein gene indicates an alternative protective mechanism contributing to longevity in Chinese individuals, using the genotype/phenotype data from 443 centenarians, 794 nonagenarians, and 1404 younger controls in Hainan and Sichuan Provinces (Liu et al., 2014). In their earlier study, they compared the distribution of the angiotensin-converting enzyme (ACE) insertion/deletion genotype and allele frequencies between 399 long-lived individuals aged 90 and older with 302 young controls and observed no association of the ACE gene insertion/deletion polymorphism with longevity in the Han Chinese population (Yang et al., 2009). The research group led by Li Jin and Xiaofeng Wang found a significant association of mitochondrial DNA haplogroups with exceptional longevity in the Chinese population based on a sample of 463 centenarians and nonagenarians, and 1389 controls aged 40-69 (Cai et al., 2009). Using genotype/phenotype data from 26 centenarians and 174 nonagenarians, the research group led by Ze Yang and Liang Sun explored the gender-based DNA methylome difference in nonagenarians/centenarians. Functional enrichment analysis of these genes revealed that DNA hypermethylation and hypomethylation may regulate genes involved in different processes or pathways, some of which may contribute to the gender gap of life span. In addition, identification of Xbased hypomethylated probes and genes could provide evidence for better understanding of the mechanism of longer lives in females (Sun et al., 2014). Another study from this group found that mtDNA haplogroup $\mathrm{F}$ is significantly associated with longevity and health in the female Chuang minority population in Southern China, based on samples of 367 long-lived individuals aged $90+$ and 371 middle-aged controls younger than 65 (Feng et al., 2011). Wangwei Cai and his research group analyzed a sample of 276 centenarians from Hainan province, and found that the polymorphism of I405V and TaqIB locus in CETP gene are significantly associated with exceptional longevity (Zhang et al., 2011).

\section{Gene-environment interactions (GxE)}

\subsection{Effects of FOXO-tea drinking interactions on cognitive impairment and mortality}

The effects of tea consumption on diseases vary by genotypes (Bonner et al., 2005; Yuan et al., 2005; Lin et al., 2012 Yuan et al., 2005; Lin et al., 2012). Belguise et al. (2007), for example, showed that intake of green tea polyphenol epigallocatechin-3Gallate (EGCG) activated FOXO3A gene expressions, which in turn induced estrogen receptor- $\alpha$ (ER- $\alpha$ ) expression and reversed the invasive phenotype of breast cancer cells in mouse. Several other studies using animal and human cell models demonstrated that intake of tea compounds activates FOXO gene expression and modulates its biological functions (e.g., Shankar et al., 2013). Based on the prior literature, the CLHLS group for the first time investigated effects of FOXO-tea drinking interactions on health and mortality at advanced ages in centenarians and nonagenarians.

Logistic regression analysis based on data from 822 Han Chinese oldest-old aged 92+ at time of first interview in 1998 (95.1\% of them died at ages $100+$ before the 2014 wave) demonstrated that interactions between carrying FOXO1A-266 or FOXO3-310 or FOXO3-292 and tea drinking at around age 60 or at present time were significantly associated with lower risk of cognitive disability at advanced ages, adjusted for various potentially confounding factors. Associations between tea drinking and reduced cognitive disability were much stronger among carriers of the genotypes of FOXO1A-266 or FOXO3-310 or FOXO3-292 compared to non-carriers, and it was reconfirmed by analysis of three-way interactions across FOXO genotypes, tea-drinking at around age 60 , and tea-drinking at the present time (Zeng et al., 2015).

The CLHLS group conducted another analysis on effects of FOXOtea drinking interactions on mortality at advanced ages based on genotypic and phenotypic data from two independent CLHLS cohorts to facilitate discovery and replication: (1) Cohort 1998 consisted of 810 oldest-old, aged $91+$ at time of first interview in 1998, followed-up in the CLHLS 2000, 2002, 2005, 2008, 2011 waves ( $94.1 \%$ of them died at ages $100+$ before the 2014 wave). (2) Cohort 2008 consisted of 1671 oldest-old aged 91+ at time of fiirst interview in 2008 and followed-up in the CLHLS 2011 wave (68.7\% of them died at ages $100+$ or survived up to ages $100+$ in the 2011 wave). These two cohorts of Han Chinese are totally independent study samples, so there is no overlap between the CLHLS participants in Cohort 1998 and Cohort 2008 and there are no familial/kinship relations among the participants within and across the cohorts. Using these data and the Cox proportional hazard model, Zeng et al. (2016b) demonstrated that interactions between carrying FOXO1A-209 genotypes and tea drinking were significantly associated with lower risk of mortality at advanced ages, adjusted for various potentially confounding factors. These significant associations were replicated in two independent Han Chinese CLHLS cohorts $(p=0.028-0.048$ in the discovery and replication cohorts, and $\mathrm{p}=0.003-0.016$ in the combined dataset). We found that associations between tea drinking and reduced mortality were much stronger among carriers of the FOXO1A-209 genotype compared to non-carriers, and drinking tea was associated with a reversal of the negative effects of carrying FOXO1A-209 minor alleles, from a substantially increased mortality risk to substantially reduced mortality risk at advanced ages. The impacts were considerably stronger among those who carry 2 copies of the FOXO1A minor alleles than those who carry 1 copy.

Based on previously reported experiments on animal and human cell models concerning FOXO-by-tea-compounds interactions, we postulate that intake of tea compounds may activate some of the FOXO gene expression, which in turn may positively affect cognitive function in the oldest-old population; we specu- 
late that tea drinking may inhibit FOXO1A-209 gene expression and its biological functions, which reduces the negative impacts of FOX01A-209 gene on longevity (as reported in the literature) and offers protection against mortality risk at oldest-old ages.

Our empirical findings imply that the health benefits of particular nutritional interventions, including tea drinking, may, in part, depend upon individual genetic profiles, and the research on the effects of genetic-nutrient interactions could potentially be useful for rejuvenation therapies in the clinic or community-based healthy aging intervention programs.

\subsection{Effects of interactions between social/behavioral factors and ADRB2 genotypes on health at advanced ages}

Statistical analyses (including structural equation models) of genotype/phenotype data from 877 CLHLS participants aged 90+ at the time of first interview in 1998 (94.4\% of them died at ages 100 + before the 2014 wave) found that, adjusted for various potentially confounding factors, carrying ADRB2-2718 or ADRB2-2719 minor alleles significantly reduced risk of negative emotion (feelings of uselessness, anxiety and loneliness) by $34 \%(\mathrm{p}<0.01)$ or $30 \%(\mathrm{p}<0.05)$, respectively. Interactions between ADRB2-2718 or ADRB2-2719 and negative emotion reduced cognitive function by $48 \%(P<0.1)$ or $59 \%(P<0.05)$, while the correlation between carrying ADRB2-2718 or ADRB2-2719 minor allele and negative emotion indirectly increased cognitive function by $16 \%$ or $4 \%$ (Zeng et al., 2013). The findings reported in this article are generally consistent with other studies which demonstrated that ADRB2 genotypes are associated with resistance to stresses, psychological disorders, and depression (e.g., Yan et al., 2007; Pandey et al., 1987). The unique contribution by the CLHLS group is the first-time finding that interactions between ADRB2-2718 and regular exercise increased cognitive function by $155 \%$ ( $\mathrm{p}<0.05$ ); interactions between ADRB22718 or ADRB2-2719 and regular exercise increased likelihood of self-reported good health by $144 \%(\mathrm{p}<0.05)$ or $211 \%(\mathrm{p}<0.01)$, respectively; and interactions between ADRB2-2719 and socialleisure activities increased likelihood of self-reported good health by $148 \%$ ( $\mathrm{p}<0.05)$ (Zeng et al., 2013).

It was discovered that the positive effects of regular exercise and social-leisure activities on cognitive function and self-reported health were much stronger among carriers of ADRB2 minor alleles compared to non-carriers; the effect of negative emotion on cognitive function was much stronger among carriers of ADRB2 minor alleles than in non-carriers. This could imply that health promotion programs (such as regular exercise and social-leisure activities) with appropriate considerations of individuals' genetic profiles would yield increased benefits at reduced costs to the programs and their participants (Zeng et al., 2013).

\section{Discussion: looking forward further more in-depth studies}

The data and research evidence reviewed in this article have demonstrated promising progress in studies on demographic and phenotypic health characteristics of centenarians as well as the effects of genetic variants and GxE interactions on healthy longevity in China. However, the results should be interpreted with caution due to inherent limitations. For example, like other GWAS studies on longevity, the major limitation of the CLHLS GWAS is a lack of physiologically relevant model systems for further biological and functional validation of the new findings. In the candidate genes and GxE studies, we only examine the associations of genetic, social/behavioral factors, and their interactions with exceptional longevity, rather than the mechanisms of how these factors work. More genotypic and phenotypic data with larger samples of all age groups among elderly population and advanced interdisciplinary research across social and biomedical sciences are called for to explore these mechanisms. More stringent tests of the significance, replication and meta-analysis studies are warranted for deeper understanding the effects of genetic variants and GxE interactions on health outcome at old ages. Another example of the limitations is evident in our FOXO-tea drinking interaction analyses. While we know that Chinese people mostly drink green teas and prior research demonstrated that consumption of green tea is associated with reduced risk of cognitive decline at ages 60 and older (e.g., Feng et al., 2010), we did not have data on what types of tea the participants drank. Furthermore, we were not able to quantify the intake of active ingredients such as EGCG and other tea catechins. Last, because of gender differences in longevity, the sub-sample size of male centenarians was not large enough so we included sex as a covariate in our publications on genetic effects and GxE interactions on healthy longevity; we have not yet published the detailed analysis for the male and female oldest-old separately, which is one of our ongoing research projects. These and other limitations inherent in our published studies on centenarians in China will need to be addressed in the near future based on forthcoming new genotypic/phenotypic datasets for all elderly age groups with much larger sample sizes for both genders.

\section{Disclaimer}

Views expressed in this paper are solely those of the authors and do not necessarily reflect those of Peking University, Duke University, National University of Singapore, the United Nations, and Max Planck Institute for Demographic Research.

\section{Acknowledgements}

The CLHLS study and Yi Zeng's work for this article are supported by the National Natural Science Foundation of China (71233001, 71490732, 71110107025) (YZ), and the US NIA/NIH (R01 AG023627, YZ; 2P01AG031719, JWV and ZY). We thank Muqi Guo very much for her excellent research assistance. We are grateful for the thoughtful comments provided by Jessica Sautter and Huashuai Chen.

\section{References}

Adams, E.R., et al., 2008. Centenarian offspring: start healthier and stay healthier. J Am. Geriatr. Soc. 56 (11), 2089-2092.

Asai, T., et al., 2002. MAP kinase signalling cascade in Arabidopsis innate immunity Nature 415, 977-983.

Balard, F., et al., 2011. Are men aging as oaks and women as reeds? A behavioral hypothesis to explain the gender paradox of French centenarians. J. Aging Res. http://dx.doi.org/10.4061/2011/371039.

Barzilai, N., et al., 2001. Offspring of centenarians have a favorable lipid profile. J. Am. Geriatr. Soc. 49, 76-79.

Baudisch, A., Vaupel, J.W., 2012. Getting to the root of aging. Science 338, 618-619.

Belguise, K., Guo, S., Sonenshein, G.E., 2007. Activation of FOXO3a by the green tea polyphenol epigallocatechin-3-gallate induces estrogen receptor $\alpha$ expression reversing invasive phenotype of breast cancer cells. Cancer Res. 67, 5763-5770.

Blazer, D.G., Fillenbaum, G., Burchett, B., 2001. The APOE-E4 allele and the risk of functional decline in a community sample of African American and white older adults. J. Gerontol. A. Biol. Sci. Med. Sci. 56, M785-M789.

Blazer, D.G., et al., 1991. The association of age and depression among the elderly: an epidemiological exploration. J. Gerontol. 46, M210-M215.

Bonafè, M., et al., 2001. A gender-dependent genetic predisposition to produce high levels of IL-6 is detrimental for longevity. Eur. J. Immunol. 31, 2357-2361.

Bonner, M.R., et al., 2005. Green tea consumption, genetic susceptibility, PAH-rich smoky coal, and the risk of lung cancer. Mutat. Res.-Gen. Toxicol. Environ. 582, 53-60.

Broer, L., et al., 2015. GWAS of longevity in CHARGE consortium confirms APOE and FOXO3 candidacy. J. Gerontol A Biol. Sci. Med. Sci. 70, 110-118.

Cai, X.-y., et al., 2009. Association of mitochondrial DNA haplogroups with exceptional longevity in a Chinese population. PLoS One, http://dx.doi.org/10. 1371 /journal.pone.0006423.

Chen, H., 2010. Assessment of the quality of the cross-sectional data collected in the 2008-2009 wave of Chinese Longitudinal Healthy Longevity Survey. In: 
Zeng, Y. (Ed.), Research on Elderly Population, Family, Health and Care Needs/Costs (in Chinese). Science Press, Beijing, pp. 350-352.

Coale, A.J., Li, S.M., 1991. The effect of age misreporting in China on the calculation of mortality rates at very high ages. Demography 28, 293-301.

Cohen, H.J., et al., 2003. Coagulation and activation of inflammatory pathways in the development of functional decline and mortality in the elderly. Am. J. Med. $114,180-187$.

Connor, K.M., Davidson, J.R., 2003. Development of a new resilience scale The Connor-Davidson resilience scale (CD-RISC). Depress. Anxiety 18, 76-82.

Deelen, J., et al., 2014. Genome-wide association meta-analysis of human longevity identifies a novel locus conferring survival beyond 90 years of age. Hum. Mol. Genet. 23, 4420-4432.

Feng, J., et al., 2011. Association of mtDNA haplogroup F with healthy longevity in the female Chuang population, China. Exp. Gerontol. 46, 987-993.

Feng, L., et al., 2010. Cognitive function and tea consumption in community dwelling older Chinese in Singapore. J. Nutr. Health Aging 14, 433-438.

Field, D., Millsap, R.E., 1991. Personality in advanced old age: continuity or change? J. Gerontol. 46, 299-308.

Goodkind, D., 2009. Review of the book Healthy Longevity in China: Demographic Socioeconomic, and Psychological Dimensions. Pop Stud. 63, 312-313.

Gu, D., Dupre, E.M., 2008. Assessment of reliability of mortality and morbidity in the 1998-2002 CLHLS waves. In: Zeng, Y., et al. (Eds.), Healthy Longevity in China: Demographic, Socioeconomic, and Psychological Dimensions. Springer Publisher, Dordrecht, The Netherlands, pp. 99-115.

Gu, D., et al., 2008. General data quality assessment of the CLHLS. In: Zeng, Y., et al (Eds.), Healthy Longevity in China: Demographic, Socioeconomic, and Psychological Dimensions. Springer Publisher, Dordrecht, The Netherlands, pp $39-60$.

Hao, Z., et al., 2016. Hair elements and healthy aging: a cross-sectional study in Hainan Island, China. Environ. Geochem. Health 38, 723-735.

Hardy, S.E., Concato, J., Gill, T.M., 2002. Stressful life events among community-living older persons. J. Intern. Med. 17, 841-847.

He, Y.-H., et al., 2014. Mitochondrial DNA content contributes to healthy aging in Chinese: a study from nonagenarians and centenarians. Neurobiol. Aging 35 1779. e1-1779. e4.

He, Y.-H., et al., 2016. Improved lipids, diastolic pressure and kidney function are potential contributors to familial longevity: a study on 60 Chinese centenarian families. Sci. Rep. 6, 21962, http://dx.doi.org/10.1038/srep21962.

Jeune, B., 1995. In search of the first centenarians. In: Jeune, B., Vaupel, J. (Eds.) Exceptional Longevity: from Prehistory to the Present. Odense University Press, Odense, Denmark, pp. 11-24

Jopp, D., Rott, C., 2006. Adaptation in very old age: exploring the role of resources, beliefs, and attitudes for centenarians' happiness. Psychol. Aging 21, 266-280.

Ju, C.A., Jones, G.W., 1989. Aging in ASEAN: Its Socio-economic Consequences. Institute of Southeast Asian Studies, Pasir Panjiang, Singapore.

Kirkwood, T.B.L., Kowald, A., 2012. The free-radical theory of ageing-older, wiser and still alive. Bioessays 34, 692-700.

Koenig, R., 2001. An island of genetic parks. Science 291, 2075-2076.

Lamb, V.L., 1999. Active Life Expectancy of the Elderly in Selected Asian Countries NUPRI Research Paper Series No. 69. Nihon University, Population Research Institute, Tokyo, Japan.

Lamond, A.J., et al., 2009. Measurement and predictors of resilience among community-dwelling older women. J. Psychiatr. Res. 43, 148-154.

Li, D., Wu, T.C.Z., 2008. An exploration of the subjective well-being of the Chinese oldest-old. In: Zeng, Y., et al. (Eds.), Healthy Longevity in China: Demographic Socioeconomic, and Psychological Dimensions. Springer Publisher, Dordrecht, The Netherlands, pp. 347-356.

Li, Y., et al., 2009. Genetic association of FOXO1A and FOXO3A with longevity trait in Han Chinese populations. Hum. Mol. Genet. 18, 4897-4904.

Li, Y., et al., 2011. Trace element concentrations in hair of healthy Chinese centenarians. Sci. Total Environ. 409, 1385-1390.

Li, Y., et al., 2012. Trace elements in fingernails of healthy Chinese centenarians. Biol. Trace Elem. Res. 145, 158-165.

Lin, I.H., et al., 2012. Smoking, green tea consumption, genetic polymorphisms in the insulin-like growth factors and lung cancer risk. PLoS One, http://dx.doi. org/10.1371/journal.pone.0030951.

Liu, et al., 2014. Absence of A673T variant in APP gene indicates an alternative protective mechanism contributing to longevity in Chinese individuals. Neurobiol. Aging 35, 935. e11-935. e12.

Naumova, E., et al., 2011. Immunogenetics of ageing. Int. J. Immunogenet. 38 , 373-381.

Newman, A.B., et al., 2010. A meta-analysis of four genome-wide association studies of survival to age 90 years or older: the Cohorts for Heart and Aging Research in Genomic Epidemiology Consortium. J. Gerontol. A Biol. Sci. Med. Sci. $65,478-487$.

Pandey, G.N., Janicak, P.G., Davis, J.M., 1987. Decreased beta-adrenergic receptors in the leukocytes of depressed patients. Psychiatr. Res. 22, 265-273.

Pascucci, M.A., Loving, G.L., 2016. Ingredients of an old and healthy life a centenarian perspective. J. Holist. Nurs. 15, 199-213.

Poon, L.W., Cheung, S.L.K., 2012. Centenarian research in the past two decades. Asian J. Gerontol. Geriatr. 7, 8-13.

Poon, L.W., et al., 1992. The Georgia centenarian study. Int. J. Aging Hum. Dev. 34, $1-17$.

Poon, L.W., et al., 2010. Understanding centenarians' psychosocial dynamics and their contributions to health and quality of life. Curr. Gerontol. Geriatr. Res. http://dx.doi.org/10.1155/2010/680657.
Poston Jr, D.L., Luo, H., 2004. Age structure and composition of the Chinese minorities in 2000 (In Chinese). Chinese Minor. Popul. 19, 9-15.

Rebeck, G.W., et al., 1994. Reduced apolipoprotein epsilon 4 allele frequency in the oldest old: Alzheimer's patients and cognitively normal individuals. Neurology $44,1513-1516$

Riolli, L., Savicki, V., Cepani, A., 2002. Resilience in the face of catastrophe: optimism, personality, and coping in the Kosovo crisis. J. Appl. Soc. Psychol. 32 1604-1627.

Ryff, C.D., Singer, B.H., 2005. Social environments and the genetics of aging: advancing knowledge of protective health mechanisms. J. Gerontol. B Psychol. Sci. Soc. Sci. 60B, 12-23 (Special Issue I).

Sebastiani, P., et al., 2012. Genetic signatures of exceptional longevity in humans. PLoS One, http://dx.doi.org/10.1371/journal.pone.0029848.

Sebastiani, P., et al., 2015. Increasing sibling relative risk of survival to older and older ages and the importance of precise definitions of aging, life span, and longevity. J. Gerontol. A Biol. Sci. Med. Sci., 1-7, http://dx.doi.org/10.1093/ gerona/glv020.

Selim, A.J., et al., 2005. Comprehensive health status assessment of centenarians: results from the 1999 large health survey of veteran enrollees. J. Gerontol. A Biol. Sci. Med. Sci. 60, 515-519.

Shankar, S., Marsh, L., Srivastava, R.K., 2013. EGCG inhibits growth of human pancreatic tumors orthotopically implanted in Balb C nude mice through modulation of FKHRL1/FOXO3a and neuropilin. Mol. Cell. Biochem. 372, 83-94.

Shen, K., Zeng, Y., 2010. The association between resilience and survival among Chinese elderly. In: Resnick, B, et al. (Eds.), The Handbook of Resilience in Aging: The Key to Successful Aging. Springer, New York, pp. 217-229.

Soerensen, M., et al., 2013. Evidence from case-control and longitudinal studies supports associations of genetic variation in APOE, CETP, and IL6 with human longevity. Age 35, 487-500.

Spada, R.S., et al., 2007. Low total cholesterol predicts mortality in the nondemented oldest old. Arch. Gerontol. Geriatr. 4, 381-384.

Sun, F., et al., 2015. Extended maternal age at birth of last child and women's longevity in the Long Life Family Study. Menopause 22, 26-31.

Sun, L., et al., 2014. Gender-specific DNA methylome analysis of a Han Chinese longevity population. BioMed. Res. Int., http://dx.doi.org/10.1155/2014/ 396727.

Tan, Q.H., et al., 2015. Epigenetic drift in the aging genome: a ten-year follow-up in an elderly cohort of twins. In: Paper Presented at the International Conference on Interdisciplinary Research on Long-term Care and Healthy Aging, May 22-23, 2015, Hangzhou, China.

Thatcher, A.R., et al., 1998. The force of mortality at ages 80-120, http://www. demogr.mpg.de/Papers/Books/Monograph5/ForMort.htm (accessed 16.09.09).

Troen, A.M., et al., 2007. Lifespan modification by glucose and methionine in Drosophila melanogaster fed a chemically defined diet. Age 29, 29-39.

United Nations, 2015. World Population Prospects: The 2015 Revision. New York: United Nations.

Van Oyen, H., et al., 2013. Gender differences in healthy life years within the EU: an exploration of the health-survival paradox. Int. J. Public Health 58, 143-155.

Wagnild, G., 2003. Resilience and successful aging: comparison among low and high income older adults. J. Gerontol. Nurs. 29, 42-49.

Wang, D., et al., 2009. Relationships between age and gender differentials in health among older people in China. Ageing Soc. 29, 1141-1154

Wang, Z., et al., 1998. Age validation of han chinese centenarians. Genus 54 $123-141$

Wang, Z., 2001. Age Validation, Demographic Characteristics and Functional Status Among Chinese Centenarians - A Population Study of Centenarians in Beijing, Hongzhou, and Chengdu. Odense University, Denmark (Ph.D dissertation).

$\mathrm{Xu}, \mathrm{S}$., Jin, L., 2008. A genome-wide analysis of admixture in uyghurs and a high-density admixture map for disease-gene discovery. Am. J. Hum. Genet. 83, 322-336.

Xu, S., et al., 2009. Genomic dissection of population substructure of Han Chinese and its implication in association studies. Am. J. Hum. Genet. 85, 762-774

Yan, L., et al., 2007. Type 5 adenylyl cyclase disruption increases longevity and protects against stress. Cell 130, 247-258.

Yang, J.K., et al., 2009. Lack of genetic association between the angiotensin-converting enzyme gene insertion/deletion polymorphism and longevity in a Han Chinese population. J. Renin Angiotensin Aldosterone. 10, $115-118$.

Yao, Y., et al., 2015. Proteasomes, Sir2, and Hxk2 form an interconnected aging network that impinges on the AMPK/Snf1-regulated transcriptional repressor Mig1. PLoS Genet., http://dx.doi.org/10.1371/journal.pgen.1004968.

Yin, Z.X., et al., 2012. Study on lifestyle and biomarkers of centenarian in longevity areas. J. Dis. Control Prev. (in Chinese) 16, 490-494.

Yuan, J.M., et al., 2005. Green tea intake, ACE gene polymorphism and breast cancer risk among Chinese women in Singapore. Carcinogenesis 26, 1389-1394.

Zeng, Y., 2012. Towards deeper research and better policy for healthy aging-using the unique data of chinese longitudinal healthy longevity survey. China. Econ. Rev. 5, 131-149.

Zeng, Y., Liu, Y.Z., George, L.K., 2003. Gender differentials of the oldest old in China. Res. Aging 25, 65-80.

Zeng, Y., Shen, K., 2010. Resilience significantly contributes to exceptional longevity. Curr. Gerontol. Geriatr. Res., http://dx.doi.org/10.1155/2010/525693.

Zeng, Y., Vaupel, J.W., 2002. Functional capacity and self-Evaluation of health and life of the oldest old in China. J. Soc. Issues 58, 733-748.

Zeng, Y., et al., 2002. Sociodemographic and health profiles of the oldest old in China. Popul. Dev. Rev. 28, 251-273. 
Zeng, Y., Gu, D., et al., 2008. Reliability of age reporting among the Chinese oldest-old in the CLHLS datasets. In: Zeng, Y., et al. (Eds.), Healthy Longevity in China: Demographic, Socioeconomic, and Psychological Dimensions. Springer Publisher, Dordrecht, The Netherlands, pp. 61-80.

Zeng, Y., et al., 2010. Effects of FOXO genotypes on longevity: a biodemographic analysis. J. Gerontol. A Biol. Sci. Med. Sci. 65, 1285-1299.

Zeng, Yi, et al., 2011. Interactions between life stress factors and carrying the APOE4 allele adversely impact self-reported health in old adults. J. Gerontol. A Biol. Sci. Med. Sci. 66A, 1054-1061.

Zeng, Y., et al., 2012. Health consequences of familial longevity influence among the Chinese elderly. J. Gerontol A Biol. Sci. Med. Sci. 68, 473-482.

Zeng, Y., et al., 2013. Interactions between Social/behavioral factors and ADRB2 genotypes may be associated with health at advanced ages in China. BMC Geriatr. 13, 91.

Zeng, Y., et al., 2015. GxE interactions between FOXO genotypes and tea drinking are significantly associated with cognitive disability at advanced ages in China. J. Gerontol. A Biol. Sci. Med. Sci. 70, 426-433.
Zeng, Y., et al., 2016a. Novel loci and pathways significantly associated with longevity. Sci. Rep. 6, 21243, http://dx.doi.org/10.1038/srep21243.

Zeng, Y., et al., 2016b. Interaction between FOXO1A-209 genotype and tea drinking is significantly associated with reduced mortality at advanced ages. Rejuvenation Res. 19, 195-203.

Zhang, Y., et al., 2011. The polymorphism of CETP gene in centenarians of Hainan province. Mod. Prev. Med. 38, 3273-3277 (In Chinese).

Zeng Y., et al. 2017. Improvements in survival and activities of daily living despite declines in physical and cognitive functioning among the oldest-old in China-Evidence from a cohort study. Forthcoming in Lancet.

Zhang, Z., 2006. Gender differentials in cognitive impairment and decline of the oldest old in China. J. Gerontol. B Psychol. Sci. Soc. Sci. 61, S107-S115.

Zhao, L., et al., 2012. Common genetic variants of the $\beta 2$-adrenergic receptor affect its translational efficiency and are associated with human longevity. Aging Cell 11, 1094-1101. 